\title{
Clinical Evaluation of Nitrofurantoin in Patients of Acute Lower Urinary Tract Infection
}

\author{
Nagma Bansal*1, VK Bajaj', Geeta Walia ${ }^{3}$ Bakhshish Singh \\ ${ }^{1}$ Department of Pharmacology, Guru Gobind Singh Medical College, Faridkot, Punjab, India \\ ${ }^{2}$ Department of Pharmacology, Government Medical College, Patiala, Punjab, India \\ ${ }^{3}$ Department of Microbiology, Government Medical College, Patiala, Punjab, India \\ ${ }^{4}$ Department of Medicine, Government Medical College, Patiala, Punjab, India \\ *Corresponding author: Nagma Bansal, Department of Pharmacology, Guru Gobind Singh Medical \\ College, Faridkot, Punjab, India, E-mail: Dr.nagma86@yahoo.com \\ Received: April 05, 2017; Revised: April 18, 2017; Published: April 22, 2017 \\ Copyright: @2017 Bansal N, et al. This is an open-access article distributed under the terms of the Creative Commons \\ Attribution License, which permits unrestricted use, distribution, and reproduction in any medium, provided the original \\ author and source are credited. The article has been previewed and authenticated by the Authors before sending the \\ publication for print. The Journal, Editor and the Editorial Board are not entitled or liable to either justify or responsible for \\ inaccurate and misleading data if any. It is the sole responsibility of the Author concerned.
}

Citation: Bansal N, Bajaj VK, Walia G, et al. Clinical Evaluation of Nitrofurantoin in Patients of Acute Lower Urinary Tract Infection. Int J Pharm Pharmacol 2017; 1: 110. doi: 10.31531/25813080.1000110

\footnotetext{
Abstract

Objective: The Urinary tract is one of the most common sources for life threatening infection. A broad range of organisms may be isolated. Various antibiotics are used for the treatment but rising resistance among pathogenic organisms is one major concern in choosing effective treatment option. The main objective of the study was to check the efficacy and safety of nitrofurantoin (100 mg BD dose) for five days in cases of acute lower UTI.

Material and Methods

Study design: open label, single arm study.

Study population: Seventy patients presenting with clinical symptoms of urinary tract infection were selected which were further confirmed by urine culture in the microbiology department.

Protocol: Urine culture and sensitivity testing for nitrofurantoin was done. After confirmation of the causative organism and sensitivity testing patients were prescribed nitrofurantoin (100 $\mathrm{mg}$ BD) for five days and were told to revisit the OPD on $7^{\text {th }}$ day. Urine culture was repeated to see the bacterial eradication rate.

Results: Sensitivity reported to nitrofurantoin is $84.3 \%$. Among all the pathogenic organism's highest sensitivity was shown by Proteus (87.5\%), followed by E. coli (84.7\%), Staphylococcus aureus $(83.3 \%)$, Klebsiella (70\%). The bacterial eradication rate is $91.5 \%$.

Keywords: Urinary tract infection, Nitrofurantoin, Resistance
}

\section{Introduction}


Urinary Tract Infection (UTI) is one of the most common bacterial infections and second most common infectious disease in the community practice [1]. All the age groups from children to geriatric are affected. It is one of the common cause of morbidity in both outpatient as well as hospitalized patients. Also, it is one of the most common nosocomial infection. The impact of the disease is even worst in developing countries due to lack of awareness of the UTIs caused by multidrugresistant (MDR) pathogens and the possibility of transfer of MDR traits between them [2,3].

Urinary tract infection is an inflammatory response of the urothelium to bacterial invasion that is usually associated with bacteriuria \& pyuria [4]. Worldwide about 150 million people are diagnosed with UTI each year, costing the global economy in excess of 6 billion US dollars. In United States only it has been estimated that it affects 8 million American women and accounts for approximately the same number of ambulatory care visits per year. The estimated direct costs are more than $\$ 1$ billion per year. Indirect costs also result from lost productivity and quality of life [5].

Escherichia coli (E. coli) is the commonest organism causing UTI. Other causative organisms include Klebsiella, Staphylococcus aureus, Staphylococcus saprophyticus, Proteus, Streptococcus faecalis, Streptococcus pyogenes, and Citrobacter [6,7].

Clinical presentation may be different in different age groups. Adults may present with features like dysuria, increase in frequency, urgency of voiding, fever, chills, rigors, headache, groin or loin pain feeling of incomplete voiding, nausea, vomiting, malaise [8-10].

As we know antimicrobial resistance is one of the major threats due to wide spread use of antimicrobial drugs in general population. Also, it is known that the common infecting organism and pattern of resistance changes over time. The agents most commonly used for the treatment of uncomplicated UTIs include the combination drug trimethoprim and sulfamethoxazole, trimethoprim, $\beta$ lactams, fluoroquinolones, nitrofurantoin, and fosfomycin tromethamine. These agents are preferred because of their safety, spectrum of activity against suspected uropathogens, and Bansal N, et al. Int J Pharm Pharmacol favorite pharmacokinetic profiles. In the treatment of UTI the resolution of bacteruria is strongly correlated with the concentration of the antimicrobial agent in the urine rather than serum levels. All the antimicrobials approved for UTI achieve inhibitory urinary concentrations that significantly exceed serum levels [11].

Resistance to nitrofurantoin remains low despite $>60$ years of use. Since this drug affects bacterial metabolism in multiple pathways, several mutational steps are required for the development of resistance. Although nitrofurantoin has traditionally been prescribed as 7-day regimen, similar microbiological and clinical efficacies are noted with a 5-day course. Most floroquinolones are highly effective for short course therapy for cystitis. Commonly used are ofloxacin, norfloxacin, ciprofloxacin and levofloxacin. The main concern regarding their use is the propagation of resistance. Nitrofurantoin has gained importance recently because it has retained activity against a useful proportion of urinary tract coliforms that have acquired resistance to trimethoprim, oral $\beta$ lactams and quinolones [12].

\section{Material and Methods}

The study was conducted in tertiary hospital in north India. After taking permission from the Institutional Ethical Committee and informed consent from the patients the project was started. Seventy patients attending medicine department were selected. Clinical diagnosis was further confirmed by urine culture in microbiology department of the institution.

Sensitivity testing for nitrofurantoin was also done on positive cultures. Patients were given $100 \mathrm{mg}$ BD dose of nitrofurantoin for 5 days and efficacy was checked by disappearance of symptoms and eradication of organism on culture.

Patients who met the following criteria were included in the study

1. Age between $18-60$ years

2. Patients with acute lower UTI

3. Patients with UTI as evaluated by clinical symptoms and further confirmed by urine culture 
Following patients were excluded from the study:

1. Patient with history of hypersensitivity to nitrofurantoin

2. Any history of pulmonary disease

3. Patients with hepatitis

4. Pregnant patients

5. Patients with history of renal damage

\section{Investigations}

Patients with signs and symptoms of UTI were subjected to following investigations-Complete Urine Examination, Urine Culture and Sensitivity. Other routine investigations like haemoglobin, total leucocyte count, differential leucocyte count was done.

\section{Results}

After confirmation of the sensitivity of pathogenic organism to nitrofurantoin patients were prescribed the drug (100 mg BD) for 5 days. They were again called on $7^{\text {th }}$ day for assessment of symptoms and urine culture was repeated. The patients who were not relieved of their clinical symptoms completely, their treatment was extended accordingly.

\section{Demographic Details}

Maximum number of patients were seen seen in age group 21-30 (60\%) and least in >50 (Figure 1).

\section{Sex Distribution}

Female constituted 60\% of total cases (Figure 2).

\section{Pathogen Distribution}

Among organisms E. coli was the most common organism constituting $65.71 \%$ of the cases followed by Klebsiella pneumoniae (14.28\%), Proteus mirabilis (11.4\%), Staphylococcus aureus (8.57\%) (Figure 3).

\section{Sensitivity to Nitrofurantoin}

The overall sensitivity to nitrofurantoin is $84.3 \%$ and the resistance reported is $15.7 \%$. Proteus mirabilis was the most sensitive organism (87.5\%) (Figure 4).

\section{Bacterial Eradication Rate}

Bansal N, et al. Int J Pharm Pharmacol
The bacterial eradication rate is $91.5 \%$ on repeat culture whereas the organism persisted in $8.5 \%$ of cases (Figure 5).

\section{Adverse Drug Effect}

The most common adverse drug reported was nausea whereas others were abdominal pain, headache and vomiting (Figure 6).

\section{Discussion}

Uncomplicated Urinary tract infections are common across the entire age spectrum. With its diverse clinical syndromes, it remains one of the most widely misunderstood and challenging infectious diseases encountered in clinical practice. However careful diagnosis and treatment results in successful resolution of infections in most instances [5].

In the current scenario of rising antibiotic resistance, when resistance to fluoroquinolones and other antibiotics used in UTI is on rise we hypothesized that nitrofurantoin might be the best choice for this indication, due to its low frequency of use and high susceptibility rate in common UTI pathogens.

In the present study, the maximum number of individuals were in age group 21-30 years i.e. $60 \%$ of the total cases. In case of gender distribution female showed predominance constituting 42 i.e. $60 \%$ of total cases.

In a study conducted by Manjunath et al. the maximum number of cases were in age group 50-79 years constituting $43 \%$ of the cases whereas in case of gender distribution female outnumber the males constituting $58.5 \%$ of total cases [1].

In another study conducted by Das RN the most common age group presenting with UTI was 41-60 years constituting $31.4 \%$ of the cases. Here also the females outnumbered the males by contributing $62.3 \%$ of total cases [13].

\section{Distribution pattern of various organisms}

In the present study, it was found that the most common organism causing UTI is E. coli contributing to $65.71 \%$ of cases followed by Klebsiella pneumonia (14.28\%), Proteus mirabilis (11.4\%), Staphylococcus aureus $(8.57 \%)$. 
In a retrospective study conducted by Priya et al. to do the survey on causative organisms and antibiotics prescribed in Tertiary care centres in UTI, it was concluded that E. coli was the commonest causative organism in both in patients as well as outpatients. The other organisms isolated from outpatients included Pseudomonas aeruginosa (3.53\%), Klebsiella (9.09\%), Staphylococcus aureus (6.06\%), Enterobacter (4.55\%) [14].

In another study conducted by Kumari et al. to see the antibiogram pattern of isolates from UTI cases in Eastern part of Nepal. Here also $E$. coli was found to be the most predominant organism $(59.0 \%)$ followed by Klebsiella (12.6\%), Pseudomonas aeruginosa (6.9\%), Acinetobacter (5.9\%), Enterococcus sp. (4.2\%) and others $(2.6 \%)$ [15].

\section{Sensitivity Pattern to Nitrofurantoin}

In the present study, the overall sensitivity reported to nitrofurantoin is $84.3 \%$ whereas the resistance rate is $15.7 \%$. In case of individual sensitivity pattern the Proteus showed the highest sensitivity (87.5\%), followed by E. coli (84.7\%), Staphylococcus aureus (83.3\%), Klebsiella (70\%).

In a study conducted by Bean et al. in London it was found that nitrofurantoin was the most active agent (94\% susceptible) against E. coli followed by gentamicin and cefpodoxime. It was concluded that that with the exception of nitrofurantoin, resistance to agents commonly used as empirical oral treatments for UTI was extremely high [16].

In another study conducted by Chulain et al. to see the antimicrobial resistance in $E$. coli associated with urinary tract infection in the west of Ireland, it was seen that most E. coli remain susceptible to nitrofurantoin $(96.7 \%)$ [17].

It was observed that results of sensitivity pattern differ in different areas but the common point among all is that it is on higher side for nitrofurantoin.

\section{Clinical Efficacy and Bacterial Eradication Rate}

In the present study, the patients who were completely cured of symptoms were $81.4 \%$ Bansal N, et al. Int J Pharm Pharmacol whereas those who showed some improvement was $18.6 \%$. The bacterial eradication rate was $91.5 \%$.

In a study conducted by Valdes et al. to see the antimicrobial and clinical efficacy of nitrofurantoin in treatment of acute lower urinary tract infection, it was seen that the bacterial eradication rate was $83.3 \%$. Urinary nitrofurantoin concentrations were mostly above minimum inhibitory concentrations of the isolated uropathogens [18].

In another study conducted by Irvani et al. to compare low dose, short course ciprofloxacin with co-trimoxazole or nitrofurantoin in the treatment of uncomplicated urinary tract infection it was seen that the bacteriological eradication 4-10 days after therapy was reported in $88 \%$ of ciprofloxacin patients, $93 \%$ of co-trimoxazole patients and $86 \%$ of nitrofurantoin patients. At the 4-6-week followup, ciprofloxacin had statistically significantly higher eradication rates $(91 \%)$ than cotrimoxazole $(79 \%$; 95\% confidence limit (CL) $=-20.6 \%, 3.9 \%)$ and nitrofurantoin $(82 \% ; 95 \%$ $\mathrm{CL}=-17.1 \%,-0.9 \%)$. Clinical resolution $4-10$ days after therapy and 4-6 week follow up was similar among the three groups [19].

\section{Adverse Drug Event}

In the present study, the adverse events reported by the patients were mild and none of the patient dropped out from the study. The most common adverse effect noted was nausea $(11.86 \%)$, followed by abdominal pain $(8.5 \%)$, headache $(6.8 \%)$, vomiting $(5.08 \%)$.

In another study conducted by Mendoza-Valdes et al. the most frequent drug related adverse event was mild headache, occurring in $10.8 \%$ of patients, two patients also discontinued the study due to rash [20].

In another study conducted by Christiaens et al. nine patients in the nitrofurantoin group reported side effects, gastrointestinal problems (4), headache (2), dizziness/fatigue (2), sleep disturbances (1) but no one discontinued the drug due to side effects [21].

In another study conducted to see the adverse reactions in children during long term antimicrobial therapy the most common 
adverse reaction associated with the use of nitrofurantoin were nausea and vomiting. But it was concluded in this study that nitrofurantoin is a safe drug for use in long term antimicrobial therapy [22].

So as evident from the above studies nitrofurantoin provides a safe and effective option for patients of acute uncomplicated urinary tract infection.

\section{Conclusion}

The conclusion of the present study is that nitrofurantoin, which is an under used antimicrobial for empiric therapy of acute lower UTI is an effective drug and the resistance acquired is very low. Moreover, the adverse events reported are very few. Another factor which goes in its favour is that this is a relatively cheaper than other drugs (like cephalosporins, floroquinolones) used to treat acute urinary tract infection. So, we can say that in our region 5-day regimen of nitrofurantoin can be safely used for treatment of acute lower urinary tract infection.

\section{References}

1. Manjunath GN, Prakash R, Annam V, et al. Changing trends in the spectrum of antimicrobial drug resistance pattern of uropathogens isolated from hospitals and community patients with urinary tract infections in tumkur and Bangalore. Int $\mathrm{J}$ Biol Med Res 2011; 2: 504-507.

2. Baral P, Neupane S, Marasini BP, et al. High prevalence of multidrug resistance in bacterial uropathogens from Kathmandu, Nepal. BMC Res Notes 2012; 5: 38.

3. Akram M, Shahid M, Khan AU. Etiology and antibiotic resistance patterns of community-acquired urinary tract infections in J N M C Hospital Aligarh, India. Ann Clin Microbiol Antimicrob 2007; 6: 4.

4. Neal DE Jr. Complicated urinary tract infections. Urol Clin North Am 2008; 35:13-22.

5. Perfetto EM, Keating K, Merchant S, et al. Acute uncomplicated UTI and E. coli resistance: implications for first-line empirical antibiotic therapy. J Manag Care Pharm 2004; 10: 17-25.

6. Lipsky BA. Urinary tract infections in men. Epidemiology, pathophysiology, diagnosis,

Bansal N, et al. Int J Pharm Pharmacol and treatment. Ann Intern Med 1989; 110: 138-150.

7. Das RN, Chandrashekhar TS, Joshi HS, et al. Frequency and susceptibility profile of pathogens causing urinary tract infections at a tertiary care hospital in western Nepal. Singapore Med J 2006; 47: 281-285.

8. Al-Otaibi FE, Bukhari EE. Clinical and laboratory profiles of urinary tract infections caused by extended-spectrum beta-lactamase-producing Escherichia coli in a tertiary care center in central Saudi Arabia. Saudi Med J 2013; 34: 171-176.

9. Juthani-Mehta M, Quagliarello V, Perrelli $\mathrm{E}$, et al. Clinical features to identify urinary tract infection in nursing home residents: a cohort study. J Am Geriatr Soc 2009; 57: 963-970.

10. Pandey KK, Vandana, Dwivedi M. Urinary tract infections and its management by renalka. The Antiseptic 2001; 98: 295-296

11. Jancel T, Dudas V. Management of uncomplicated urinary tract infections. West J Med 2002; 176: 51-55.

12. Gupta K, Traunter BW. Urinary tract infections, pyelonephritis and Prostatitis. In: Fauci AS, Braunwald E, Kasper DL, et al.editors. Harrisons Principles of Internal Medicine $18^{\text {th }}$ edn. USA: Mcgraw Hill Companies 2008; 2393-2395.

13. Das RN, Chandrashekhar TS, Joshi HS, et al. Frequency and susceptibility profile of pathogens causing urinary tract infections at a tertiary care hospital in western Nepal. Singapore Med J 2006; 47: 281-285.

14. Priya P, Radha K, Jennifer G. Urinary tract infections: a retrospective survey of causative organisms and antibiotics prescribed in a tertiary care setting. Ind $\mathbf{J}$ of Pharmacol 2002; 34: 278-280.

15. Kumari N, Ghimire G, Magar JK, et al. Antibiogram pattern of isolates from UTI cases in Eastern part of Nepal. Nepal Med Coll J 2005; 7: 116-118.

16. Bean DC, Krahe D, Warehem DW. Antimicrobial resistance in community and nosocomial Escherichia coli urinary tract isolates, London 2005-2006. Ann Clin Microbiol Antimicrob 2008; 7: 13.

17. Chulain MN, Murray AM, Feeney GC, et al. Antimicrobial Resistance in E. coli associated with urinary tract infection in the 
west of Ireland. Ir J Med Sci 2005; 174: 69.

18. Mendoza-Valdes A, Rosete A, Rios Buene $E$, et al. Nitrofurantoin in the treatment of acute lower urinary tract infections in adults. Med Klin 2010; 105: 698.

19. Irvani A, Klimberg I, Briefer C, et al. A trial comparing low dose, short course ciprofloxacin and standard 7-day therapy with co-trimoxazole or nitrofurantoin in the treatment of uncomplicated urinary tract infection. J Antimicrob Chemother 1999; 43: 67-75.

20. Mendoza-Valdes A, Rosete A, Rios Buene E, Frentzel A, Trapp S, Kirch W, Mueller
EA. Antimicrobial and clinical efficacy of nitrofurantoin in the treatment of acute lower urinary tract infections in adults. Med Klin 2010; 105: 698.

21. Christiaens TCM, De Meyere M, Verschragen $G$, et al. Randomized controlled trial of nitrofurantoin versus placebo in the treatment of uncomplicated urinary tract infection in adult women. $\mathrm{Br} \mathrm{J}$ Gen Pract 2002; 52: 729-734.

22. Uhari M, Nutinen M, Turtinen J. Adverse reactions in Children during Long term antimicrobial Therapy. Pediatr Infect $\mathbf{J}$ 1996; 15: 404-408.

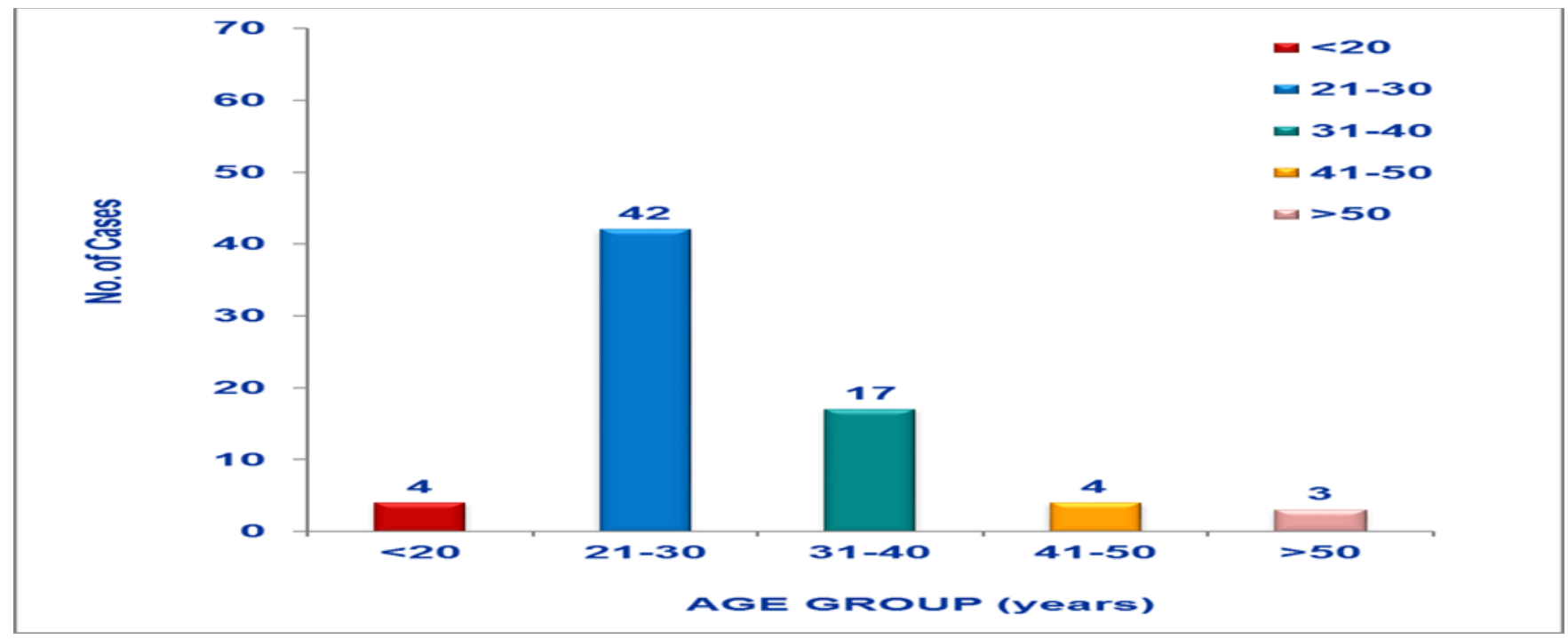

Figure 1: Bar diagram showing age-wise distribution of total cases

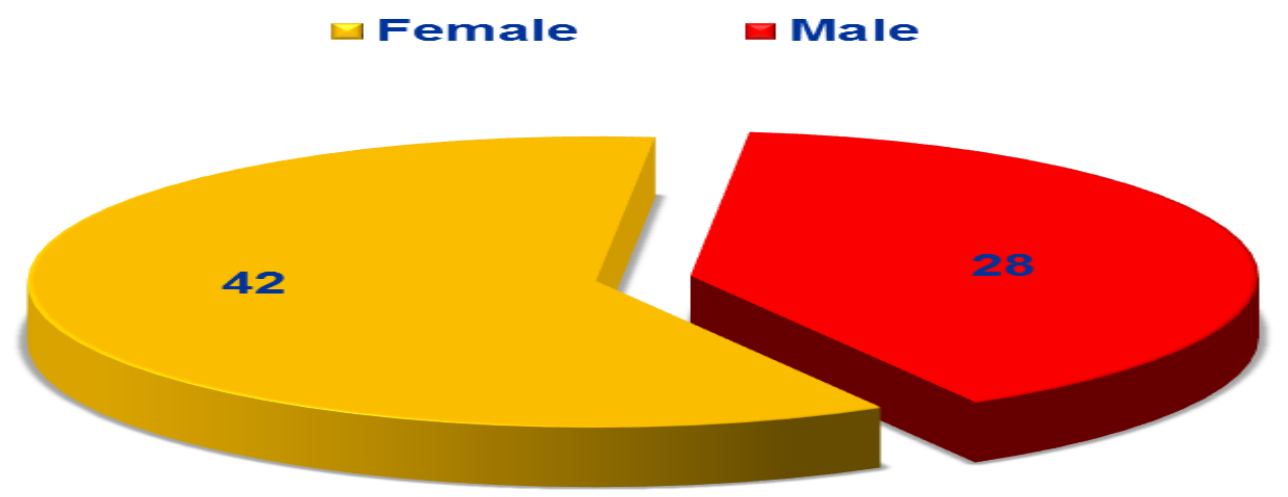

Figure 2: Pie chart showing gender wise distribution of total cases 


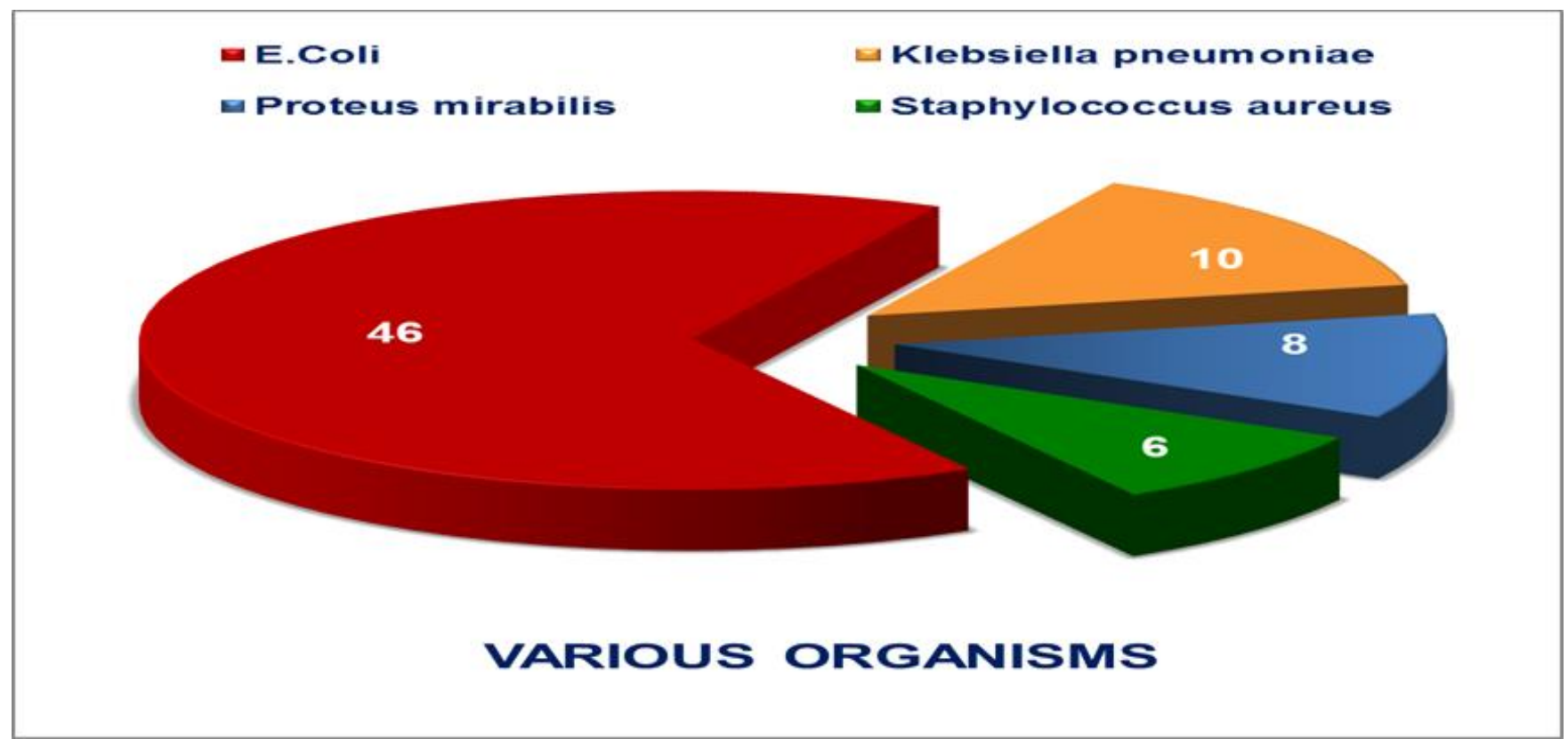

Figure 3: Pie chart showing distribution of various organisms

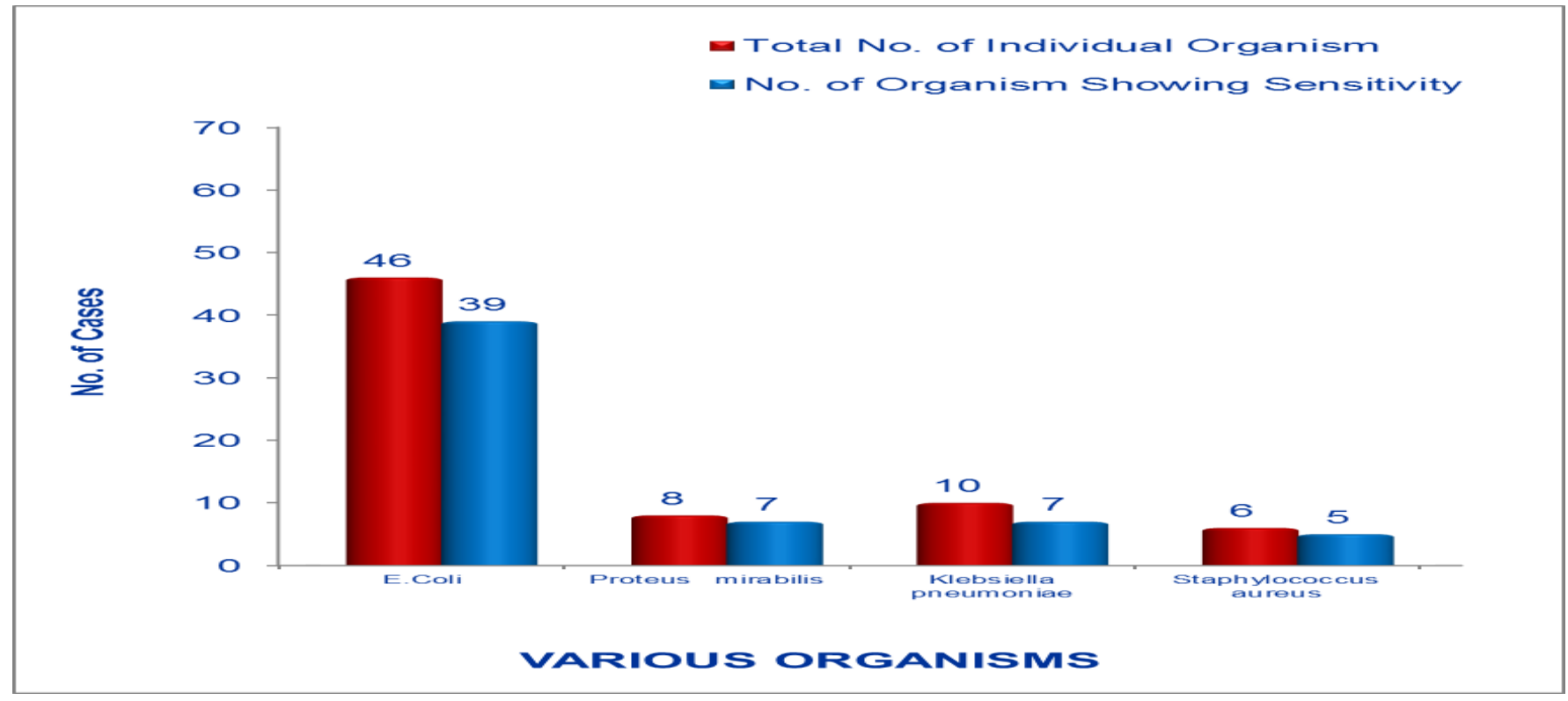

Figure 4: Bar diagram showing sensitivity pattern of various organisms to nitrofurantoin 
$\square$ No Growth $\quad$ Positive Growth

54

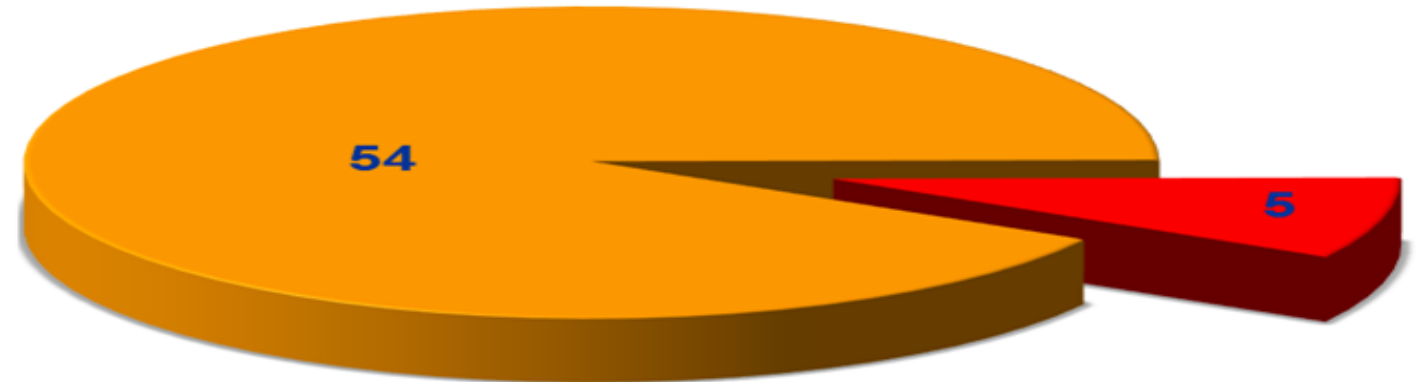

Figure 5: Pie chart showing efficacy of nitrofurantoin in terms of bacterial eradication on repeat urine culture

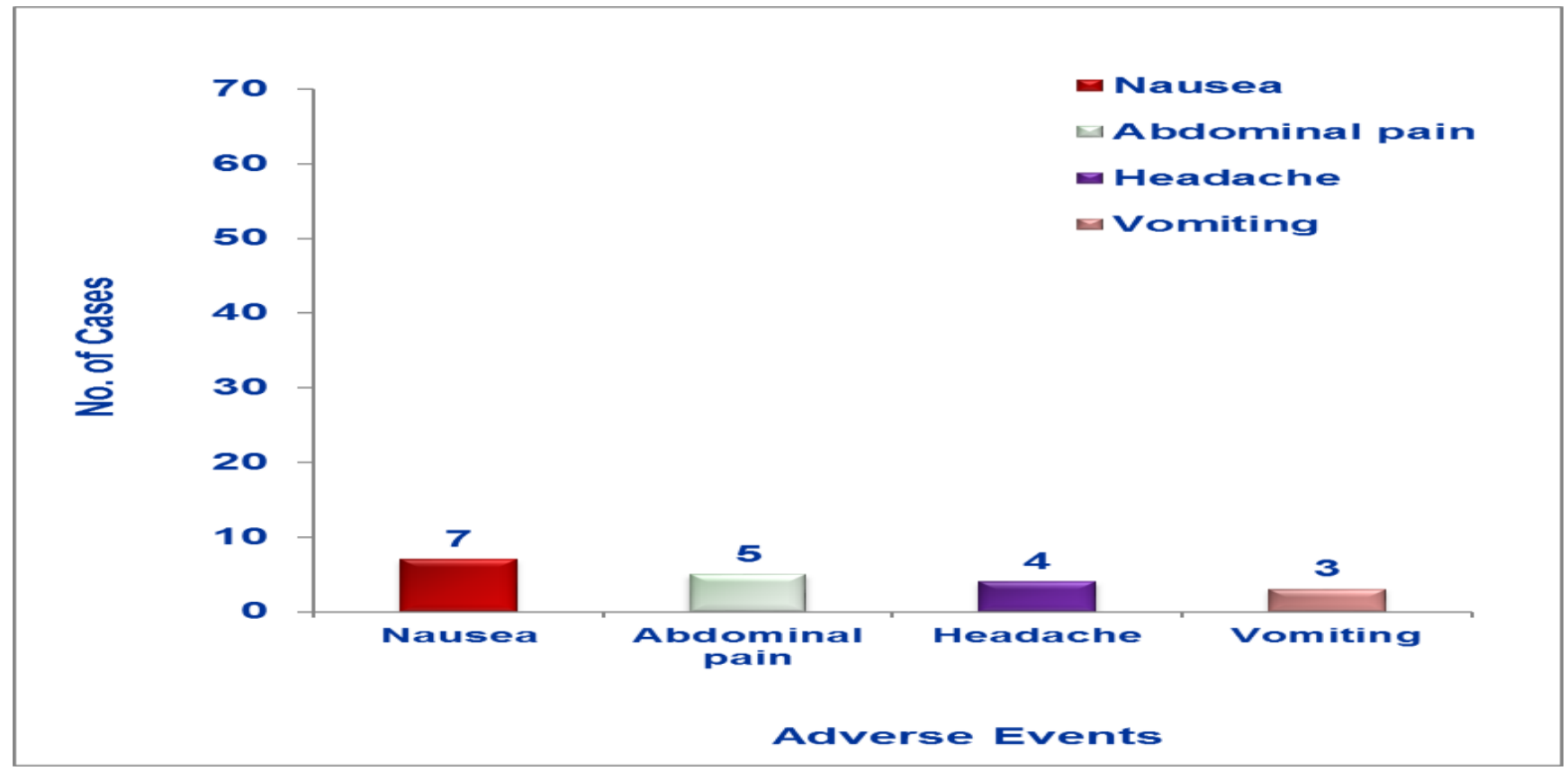

Figure 6: Bar diagram showing distribution of various drug related adverse events 
This manuscript was peer-reviewed

Mode of Review: Single-blinded

Editor: Dr. Juliana F. Roos

International Journal of Pharmaceutics and Pharmacology is an open access, peer reviewed journal published by Edwiser International.

Submit your valuable manuscript at-

editor.ijpp@edwiserinternational.com

submit.manuscript@edwiserinternational.com

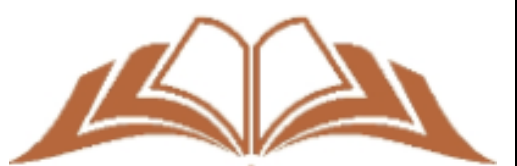

EDW $\mathrm{D}$ S E R

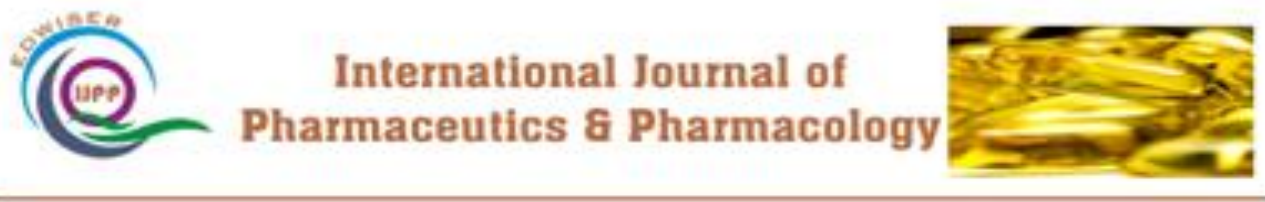

International Journal of General Medicine \& Surgery
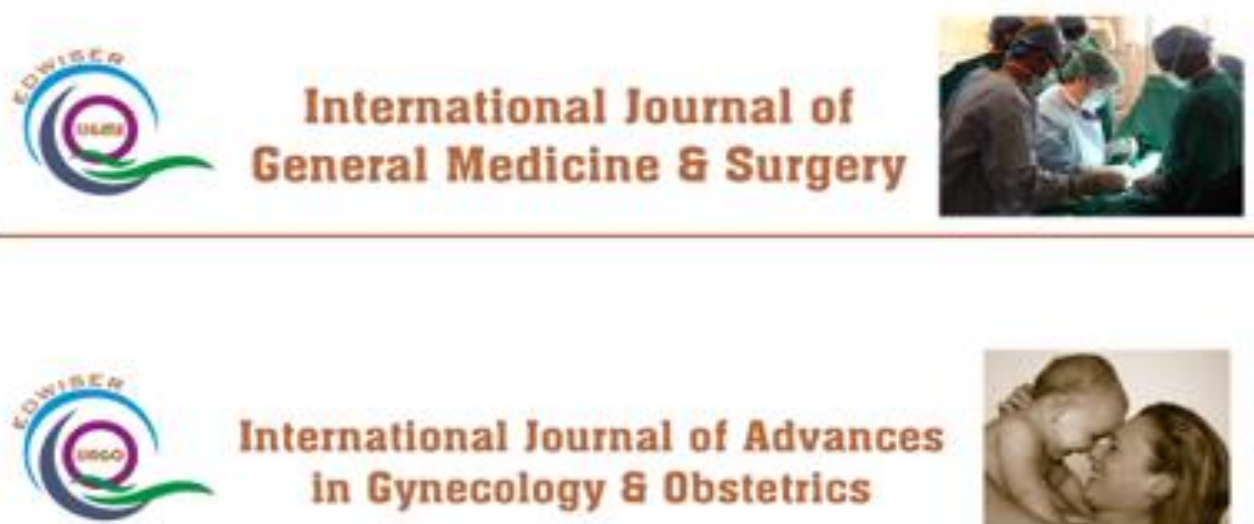

International Journal of Advances

in Gynecology \& Obstetrics
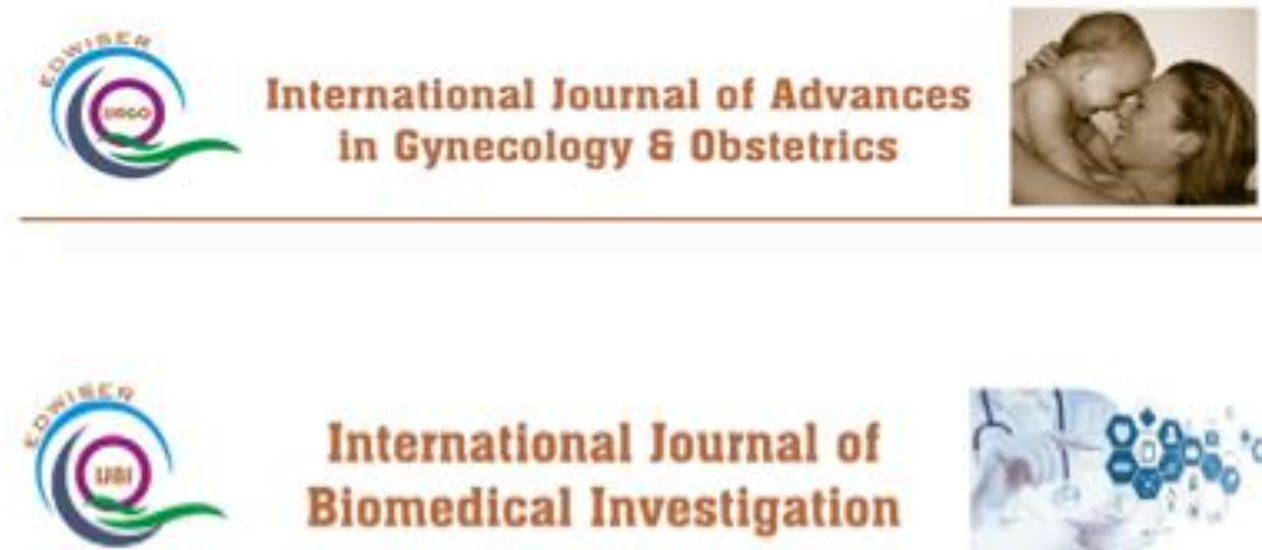

International Journal of Biomedical Investigation
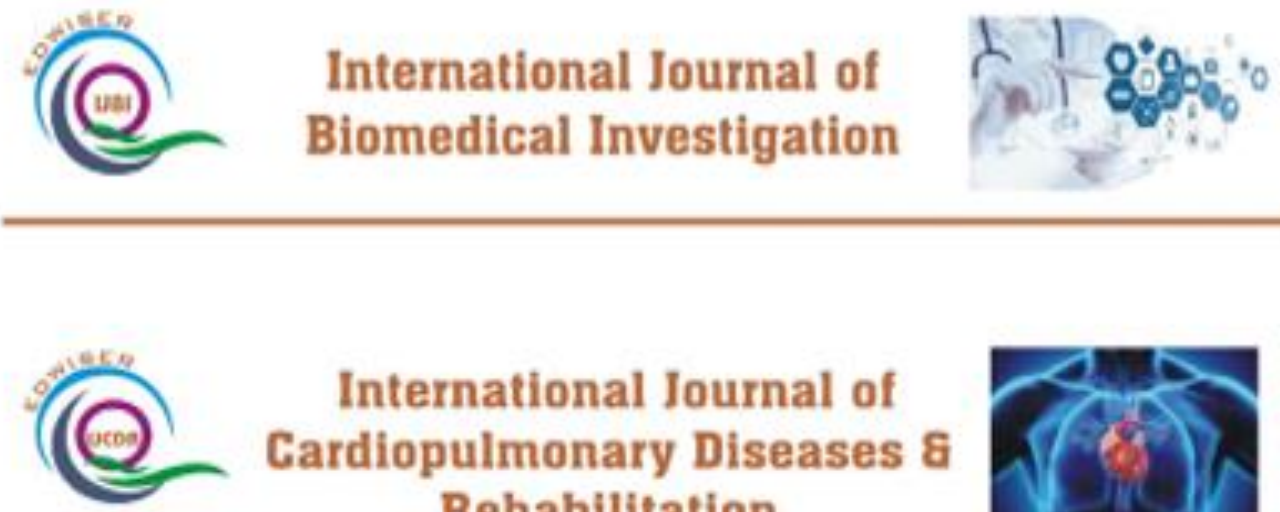

International Journal of Cardiopulmonary Diseases \& Rehabilitation

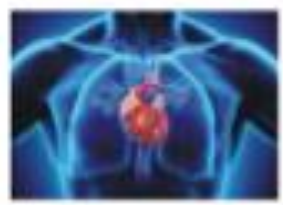

